\title{
Assisted heterogeneous multinucleation and bubble growth in semicrystalline ethylene-vinyl acetate copolymer/expanded graphite nanocomposite foams: Control of morphology and viscoelastic properties
}

\author{
O. Yousefzade, F. Hemmati, H. Garmabi , M. Mahdavi
}

Department of Polymer Engineering and Color Technology, Amirkabir University of Technology, No. 424, Hafez Ave., Tehran, Iran

\begin{abstract}
Nanocomposite foams of ethylene-vinyl acetate copolymer (EVA) reinforced by expanded graphite (EG) were prepared using supercritical nitrogen in batch foaming process. Effects of EG particle size, crosslinking of EVA chains and foaming temperature on the cell morphology and foam viscoelastic properties were investigated. EG sheet surface interestingly provide multiple heterogeneous nucleation sites for bubbles. This role is considerably intensified by incorporating lower loadings of EG with higher aspect ratio. The amorphous and non-crosslinked domains of EVA matrix constitute denser bubble areas. Higher void fraction and more uniform cell structure is achieved for non-crosslinked EVA/EG nanocomposites foamed at higher temperatures. With regard to the structural variation, the void fraction of foam samples decreases with increasing the EG content. Storage and loss moduli were analyzed to study the viscoelastic properties of nanocomposite foams. Surprisingly, the foaming process of EVA results in a drastic reduction in loss and storage moduli regardless of whether the thermoplastic matrix contains EG nanofiller or not. For the EVA/EG foams with the same composition, the nanocomposite having higher void fraction shows relatively lower loss modulus and more restricted molecular movements. The study findings have verified that the dynamics of polymer chains varies after foaming EVA matrix in the presence of EG.
\end{abstract}

Keywords: nanocomposites, foam, expanded graphite, morphology, viscoelastic properties

\section{Introduction}

The creation of polymeric cellular structures using environmentally friendly physical blowing agents in the supercritical or nonsupercritical state has been of significant interest in the past decades. Nitrogen and carbon dioxide have been applied in the polymeric foaming process as environmentally friendly blowing agents. An overview on the carbon dioxide and nitrogen assisted foaming process of glassy polymers is provided by Fleming and Kazarian [1] and Almanza et al. [2]. A literature review of polymeric foams shows that there are many studies on the preparation of high-performance polymeric foams by increasing the number of cells (bubbles) and decreasing cell size. To seek this aim, different continuous and discontinuous process variants have been developed to prepare microcellular foam morphologies from glassy polymers $[1,3,4]$. Similarly, there are some reports about clay reinforced nanocomposite foams based on semicrystalline polymers [5-8]. Moreover, microcellular foaming process is one of the techniques that have been studied for this purpose [9-16]. By rapid pressure quenching [17], melt blending using polymers with high glass tran-

\footnotetext{
*Corresponding author, e-mail: garmabi@aut.ac.ir

(C) BME-PT
} 
sition temperature $\left(T_{\mathrm{g}}\right)[4]$ and incorporating nanoclay into polymers [7], micron or submicron size cellular structures have been successfully obtained. Most polymers investigated in the previous researches have been amorphous, though there are several papers discussing microcellular foams of semicrystalline polymers $[15,16,18-23]$.

The influences of nanofiller characteristics such as aspect ratio and surface chemistry on the properties of solid polymer nanocomposites have been extensively studied [24-27]. However, the behavior of polymer composite foams is more complex than that of solid composites. The interactions between filler surface, bubbles and matrix chains in pairs and their effects on the properties of composite foams have not been fully understood. In addition, the characteristics of one component may affect the properties of the others. Therefore, to obtain polymer nanocomposite foams with optimal properties such as high electromagnetic wave shielding effectiveness or strength, a better understanding of the interactions between nanofillers, bubbles and matrix chains in pairs is an essential prerequisite. The impact of processing methods on the nanocomposite foam morphology has been examined while the aspect ratio changes of nanofillers due to the alterations of processing variables have not been considered $[12,28]$. The filler aspect ratio most likely changes the foam morphology that requires to be explored.

For polymeric foaming processes using supercritical fluids, the most controllable parameters are processing variables such as sorption pressure and temperature [15]. The influence of the sorption pressure on the foam morphology has been studied at a constant sorption temperature. Besides, diffusivity of the supercritical fluid can be highly influential [15]. Furthermore, in the case of polymer nano/microcomposites, the filler characteristics like size, aspect ratio, geometric shape and filler/polymer interaction as well as the used fluid/gas are crucially important because the bubble size, shape and formation mechanism are greatly impacted by these factors $[28,29]$.

In addition, dynamic mechanical thermal analysis (DMTA) has emerged as one of the most powerful tools available for viscoelastic property study of plastic materials. Since all polymers are viscoelastic in nature, this analytical method suits perfectly to evaluate the complex phenomena presented by polymeric materials including the main chain and side-groups motions and, in particular, different relaxations of polymer chains [30]. As a result, this turns it into a powerful technique for characterizing different polymer structures such as plastic foams. Along with this advantage, there is a well-known relationship between the damping capabilities of a certain polymer and the dynamic mechanical responses that can be also investigated by DMTA [31]. Thus this method has been applied in the present work.

Because there are few published researches on semicrystalline polymer foams containing expanded graphite (EG), microcellular nanocomposite foams of semicrystalline ethylene-vinyl acetate copolymer (EVA) and EG were chosen as the subject of this study. These foams possessing a wide range of relative density and varied cellular structures have been obtained and the influences of filler particle size, crosslinking of EVA chains and foaming temperature as a processing parameter on the nanocomposite foam morphology, void fraction and the semicrystalline polymer viscoelastic properties have been evaluated. Different loadings of EG below and above the electrical and rheological percolation thresholds have been used to show the properties of solid and foamed nanocomposites. Efforts have been particularly made to show that the damping capability of nanocomposite is reduced by changing the microstructure to foam and molecular movements are retarded even more for the nanocomposite foams with larger void fractions. Moreover, for the first time, to the best of our knowledge, the effects of EG particle size on the foaming behavior of EVA-based nanocomposites have been researched and the heterogeneous multinucleation role of EG nanosheets has been verified. Our findings show that by incorporating large EG nanosheets at small loadings, i.e. less than $5 \mathrm{wt} \%$, into non-crosslinked EVA and foaming these nanocomposites at high temperatures, very light EVA nanocomposite foams with void fractions about $70 \%$ can be obtained. In addition, for these nanocomposite foams, the optimal cell morphology with highest cell density and broadest cell size distribution is attained.

\section{Experimental section \\ 2.1. Materials}

Two types of expanded graphite powder having a sheet-like structure and different aspect ratio were 
prepared by intensely heating of graphite intercalation compound (GIC) at $1000^{\circ} \mathrm{C}$ for $30 \mathrm{sec}$. After heating, these powders were sieved. These EGs are EX100 and EX1500 grades (purchased from Ito Kokuen Co., Mie, Japan), which have an average sheet size of 149 and $2 \mu \mathrm{m}$, respectively. Since the thickness of graphite sheets are in the range of a few nanometers, EX100 has larger aspect ratio and the other one is the smaller aspect ratio EG used in this work. In our previous study, however, the morphology of heated and sieved expanded graphites was investigated before melt-compounding process by using microscopic observations. The SEM micrographs of pure expanded graphites showed that EX100 has much larger aspect ratio than EX1500. In addition, the morphology of EVA/EG nanocomposites was also evaluated and it was found that the graphite aspect ratio in the nanocomposite filled with EX100 is much larger than the nanocomposite containing EX1500 regardless of EG loading. However, the two-step melt process that was employed for melt-compounding of EVA and graphite filler has consisted of a vigorous elogational and shear flow. These flow fields have reduced the graphite sheet size. Despite this fact, the nanocomposites containing EX100 have larger aspect ratio sheets. The observed morphologies for the prepared nanocomposites have been delaminated and intercalated. Intercalation of graphite stacks has been completely obvious in the micrographs of nanocomposites having different loadings of EX100 and EX1500 graphites (refer to [30] for more information).

A commercial grade of ethylene vinyl acetate copolymer with $19 \mathrm{wt} \%$ vinyl acetate comonomer (VAc) content (Seetec VS430) was purchased from Hyundai Co. (Sosan-gun, South Korea). One of the common crosslinking agents of EVA thermoplastic is peroxide chemicals, such as dicumyl peroxide (DCP) that was supplied by Merck KGaA, Darmstadt, Germany.

\subsection{Sample preparation}

Details of solid nanocomposite preparation were presented in our previous study [30]. The aforementioned EGs with different sizes were used to obtain the nanocomposites. For producing microcellular nanocomposite foams, the sheets of specimens with thickness of $1 \mathrm{~mm}$ were molded at $110^{\circ} \mathrm{C}$ and then batch foaming process was conducted in a highpressure system. Samples were placed in the highpressure vessel, then $\mathrm{N}_{2}$ gas was fed to the pressure vessel. The vessel was kept at a constant pressure. Besides the whole system was kept at a constant temperature of $85^{\circ} \mathrm{C}$ for $4 \mathrm{~h}$ to allow nitrogen gas to saturate the sample and attain the equilibrium state. Next, the samples were foamed at temperatures above and below the crystallization temperature of EVA matrix, i.e. at 70 and $50^{\circ} \mathrm{C}$ respectively. After

Table 1. Sample codes with different formulations and processing conditions

\begin{tabular}{|c|c|c|c|c|c|}
\hline Sample code & Crosslinking state & $\begin{array}{c}\text { EG loading } \\
\text { [wt } \%]\end{array}$ & $\begin{array}{c}\text { EG size } \\
{[\mu \mathrm{m}]}\end{array}$ & Foaming state & $\begin{array}{c}\text { Foaming temperature } \\
{\left[{ }^{\circ} \mathbf{C}\right]}\end{array}$ \\
\hline EVA & non-crosslinked & 0 & - & unfoamed & $\begin{array}{ll}- \\
-\end{array}$ \\
\hline EV6EG149 & non-crosslinked & 6 & 149 & unfoamed & - \\
\hline EV6EG2 & non-crosslinked & 6 & 2 & unfoamed & - \\
\hline CEV6G149 & crosslinked & 6 & 149 & unfoamed & - \\
\hline EVFT50 & non-crosslinked & 0 & - & foamed & 50 \\
\hline EVxEG149FT50 & non-crosslinked & variable $^{*}$ & 149 & foamed & 50 \\
\hline EV6EG149FT50 & non-crosslinked & 6 & 149 & foamed & 50 \\
\hline EVxEG2FT50 & non-crosslinked & variable ${ }^{*}$ & 2 & foamed & 50 \\
\hline EV6EG2FT50 & non-crosslinked & 6 & 2 & foamed & 50 \\
\hline EV15EG2FT50 & non-crosslinked & 15 & 2 & foamed & 50 \\
\hline EVxEG149FT70 & non-crosslinked & variable $^{*}$ & 149 & foamed & 70 \\
\hline EV4EG149FT70 & non-crosslinked & 4 & 149 & foamed & 70 \\
\hline EV6EG149FT70 & non-crosslinked & 6 & 149 & foamed & 70 \\
\hline EV15EG149FT70 & non-crosslinked & 15 & 149 & foamed & 70 \\
\hline EV6EG2FT70 & non-crosslinked & 6 & 2 & foamed & 70 \\
\hline CEVxEG149FT50 & crosslinked & variable $^{*}$ & 149 & foamed & 50 \\
\hline CEV6EG149FT50 & crosslinked & 6 & 149 & foamed & 50 \\
\hline CEVxEG2FT50 & crosslinked & variable ${ }^{*}$ & 2 & foamed & 50 \\
\hline CEV6EG149FT70 & crosslinked & 6 & 149 & foamed & 70 \\
\hline
\end{tabular}

${ }^{*}$ The EG loading for these samples is variable in the range of 0 to $30 \mathrm{wt} \%$. 
the saturation of sample at a constant pressure of 11.5 $\mathrm{MPa}$, the pressure was then rapidly released and the resultant foam morphology was fixed by cooling water.

In the remaining parts, the abbreviated codes will be used for the obtained samples according to different parameters, which are summarized in Table 1.

In the abbreviated codes, C, EV, EG and FT respectively stand for crosslinked, EVA, expanded graphite and foaming temperature. If the letter ' $\mathrm{C}$ ' is used before the code, it will show that the EVA matrix was chemically crosslinked. The numbers used before and after the letter 'EG' respectively show the EG loading and sheet size. The foaming temperature, $50^{\circ} \mathrm{C}$ or $70^{\circ} \mathrm{C}$, is a number after ' $\mathrm{FT}$ '. Besides, for the solid samples, unformed ones, 'FT' will be omitted from the code like EV6G2. Similarly, for the samples that do not contain EG, 'EG' letter is not included in the codes.

\subsection{Characterization methods}

Scanning electron microscopy (SEM) of EVA/EG nanocomposite foams were performed on the cryofractured surfaces using a Seron Technologies Inc. Scanning Electron Microscope (Gyeonggi-do, Korea). The samples were subjected to brittle fracture in liquid nitrogen, and then the fractured surfaces were gold sputtered prior to the examination. Bubble size and Bubble size distribution were measured using Image Pro plus software (Media Cybernetics Inc., USA). Dynamic mechanical thermal characteristics of the obtained foam samples with thickness of 1-2 mm were evaluated using a DMTA analyzer (Triton Co. Lincolnshire, UK) under tension mode. The temperature sweep measurements were carried out over the temperature range of -50 to $50^{\circ} \mathrm{C}$, at a frequency of $1 \mathrm{~Hz}$ and a heating rate of $2^{\circ} \mathrm{C} / \mathrm{min}$. For determination of the void fraction, densities of the obtained foams were measured by solvent displacement method following the standard of ASTM D792. The void fraction of the foam samples is calculated according to ASTM D2734 by using Equation (1):

$V_{\mathrm{f}}=\frac{d_{\mathrm{u}}-d_{\mathrm{f}}}{d_{\mathrm{u}}}$

where $V_{\mathrm{f}}, d_{\mathrm{u}}$ and $d_{\mathrm{f}}$ are the void fraction of foam, density of unfoamed and foamed nanocomposites, respectively.

\section{Result and discussion \\ 3.1. Morphology}

The foaming process of nitrogen-swollen polymer occurs after destabilizing the thermodynamic equilibrium state, which is induced by quenching the pressure here. During the pressure quenching, the polymer mixture becomes supersaturated with nitrogen gas, which leads to cell nucleation and growth. Depending on the melting temperature or glass transition temperature of polymer, the matrix can vitrify, thereby suppressing cell growth. However, for EVA copolymer as matrix, only the melting temperature is required to be considered, since the glass transition temperature is far below the processing temperatures.

\subsubsection{Effects of EG particle size}

One of the parameters, which determines the cell morphology of a nanocomposite foam, is the dispersion and delamination state of nanofiller in the polymer matrix of solid nanocomposite before any foaming process. In our previous study [30], the effect of two-step melt mixing process on the dispersion of EG particles in EVA copolymer matrix was investigated. As mentioned, better dispersion state of nanofiller in polymer matrix changes the foam morphology significantly and leads to higher cell density and narrower cell size distribution. In a similar manner, the cell size and density are considerably related to the filler loading on account of the profound impact of particle content on the dispersion state, thereby affecting the accessible solid surface area for cell heterogeneous nucleation. In other words, the nanofiller aggregation is decreased at lower particle loadings, whereupon the efficiency of fillers in providing nucleation sites and foaming will improve. The effect of EG content on the foam morphology is shown in Figure 1. More bubbles in the nanocomposite foam sample filled with $15 \mathrm{wt} \%$ of EG are seen. Measured cell size and cell size distribution were presented in Table 2. The average cell size of EV15EG149FT70 sample is lower than the one for EV4EG149FT70 foam. At higher filler loading approximately equal to the EG percolation threshold, more aggregates of particles remain unchanged and consequently, worsen the performance of EG particle surface in cell nucleation process, thus the filler effectiveness in this process is weakened lower than that is expected. The aggregates of graphite at higher loading can be observed in Figure $1 \mathrm{~b}$. 


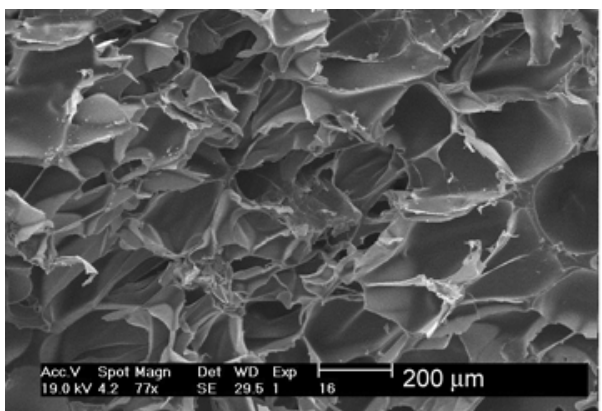

a)

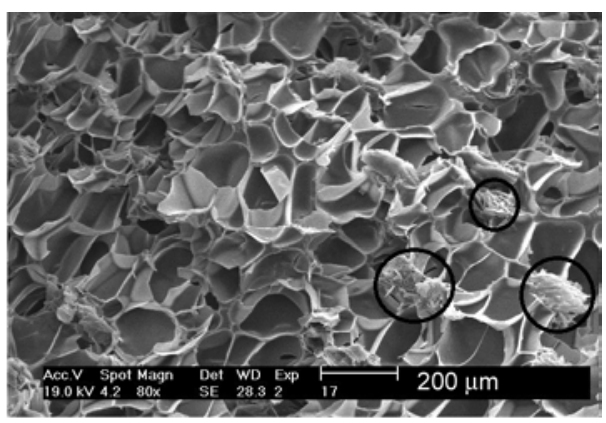

b)

Figure 1. SEM micrographs of EVA/EG nanocomposite foams: (a) EV4EG149FT70 and (b) EV15EG149FT70; effect of EG loading on cell morphology. The black circles show some of graphite aggregates.

According to the nucleation theory, the classical theory is an approach that is currently used to describe the bubble nucleation in polymer foams, although its prediction on the nucleation rate can be easily off by several orders of magnitude. Among the polymer foaming community, the discrepancy between the classical theory and experimental results is sometimes attributed to the intervening heterogeneous nucleation provided by filler solid surface and has led to the classical theory modifications by incorporating certain aspects specific to the polymer foaming process [9, 32-34]. Nanoparticles have been validated that undoubtedly serve with heterogeneous nucleation sites and their influence on the cell density has been qualitatively represented by the classical nucleation theory $[32,34]$. In heterogeneous nucleation process, the highest nucleation efficiency can be achieved only if the nucleation on the nucleant surface is energetically favored comparing with its homogeneous counterpart and the nucleant is well dispersed in the polymer matrix. In most cases, the measured cell density is much lower than the potential nucleant density, implying that either the nucleant surface is not energetically favored, or the dispersion state of nucleant is not good.

In addition, the aspect ratio of filler is another determinant of particle effectiveness in heterogeneous

Table 2. Average cell size and the standard deviation of cell size for different EVA/EG foams

\begin{tabular}{|l|c|c|c|}
\hline \multicolumn{1}{|c|}{ Sample } & $\begin{array}{c}\text { Average } \\
\mathbf{c e l l} \text { size } \\
{[\boldsymbol{\mu \mathbf { m } ]}}\end{array}$ & $\begin{array}{c}\text { Standard } \\
\mathbf{d e v i a t i o n} \\
{[\boldsymbol{\mu \mathbf { m } ]}}\end{array}$ & $\begin{array}{c}\text { Standard } \\
\mathbf{d e v i a t i o n} \\
{[\mathbf{\%}]}\end{array}$ \\
\hline EV4EG149FT70 & 138 & 52 & 37 \\
\hline EV15EG149FT70 & 79 & 30 & 37 \\
\hline EV6EG2FT50 & 23 & 11 & 47 \\
\hline EV6EG149FT50 & 34 & 21 & 61 \\
\hline CEV6EG149FT70 & 96 & 55 & 57 \\
\hline EV6EG149FT70 & 112 & 57 & 50 \\
\hline
\end{tabular}

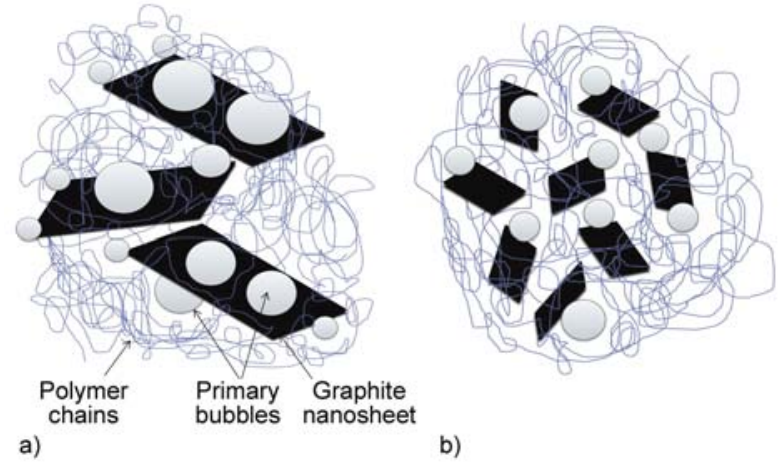

Figure 2. A schematic indicating the possible nucleation mechanism of EG particles: (a) each EG particle provides multiple nucleation sites; (b) each EG particle acts as a single nucleation site

nucleation process. As the filler aspect ratio increases, a single particle may shift from nucleating one bubble to simultaneously initiating multiple bubbles [29]. Therefore, the number of possible heterogeneous nucleation sites is most probably higher than the number of expanded graphite sheets owing to the potential for sidewall nucleation, which is schematically shown in Figure 2. The possibility of this happening is higher for larger nanosheets. This phenomenon provides a proof that for the nanocomposites filled with expanded graphite with high particle size or aspect ratio, more than one heterogeneous nucleation site is likely to be accessible along a given expanded graphite nanosheet.

As shown in Figure 3, different cell morphologies can be realized for the nanocomposite foams filled with EX100 and EX1500. Higher cell density and broader cell size distribution of sample filled with EX100 give evidence for stronger multinucleation role of EX100, which stems from larger solid surface area available for heterogeneous bubble nucleation (See the results of EV6EG2FT50 and EV6EG149FT50 samples in Table 2). Furthermore, it can be discerned in this figure that many cells are grown and 


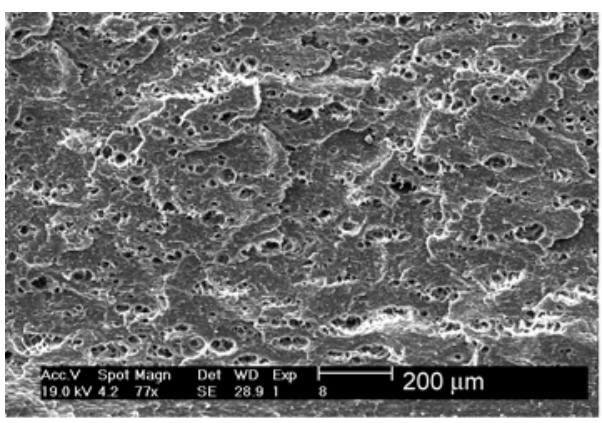

a)

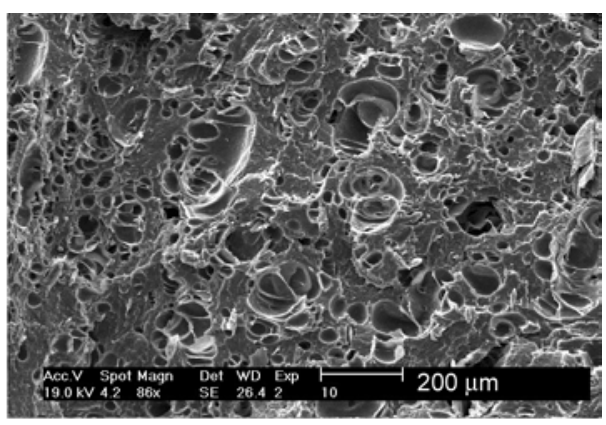

b)

Figure 3. SEM micrographs of nanocomposite foams: (a) EV6EG2FT50 and (b) EV6EG149FT50; effect of EG particle size

impinged into each other especially in the foam sample containing EX100. According to Table 2, as it is expected, the heterogeneity of bubble size in the sample containing EX100 (EV6EG149FT50) is much more than the sample containing EX1500 (EV6EG2FT50).

\subsubsection{Effects of EVA crosslinking}

Another influential factor in cell evolution of polymeric foams is the polymer properties as matrix. Molecular weight and distribution, chemical and physical architecture of polymer chains, and the fluidity of polymer are substantially important to determine the cell nucleation and growth behavior [23]. Besides, the melt strength of polymer is another notable key to characterize the foams properties. For EVA copolymer as a thermoplastic matrix, the crosslinking of polymer chains is the best way to increase the melt strength. Therefore, in this study the crosslinking and foaming process of solid nanocomposites were completed in two separate steps, different from other investigations [35-37]. First, the chemical crosslinking of EVA copolymer macromolecules was carried out in the compression molding process. Second, the cured samples were foamed in the autoclave at the same conditions mentioned before. The differences in cell morphologies of cured and noncured foam samples are shown in Figure 4. Considering the fact that all foam samples were foamed under the same conditions, more nonfoamed regions of cured samples demonstrate that the presence of chemical crosslinks among polymer chains affects both cell nucleation and cell growth stages of foaming process. These stages are influenced respectively by lowering the gas diffusivity, i.e. causing insufficient nitrogen saturation state, and intensifying the local melt flow index of polymer. The results that are gathered in Table 2 show that CEV6EG149FT70 sample has much lower average cell size than EV6EG149FT70 foam.

\subsubsection{Effects of foaming temperature}

As mentioned earlier, along with the material parameters, the processing ones such as the foaming pressure and temperature also impact on the cell morphology and thereby, changing the foam physical and mechanical properties. In accordance with the classical nucleation theory, the cell density and the bubble shape are affected by the foaming pressure or pressure sharp drop during foaming process. In all related researches, it has been demonstrated that the number of cells per unit volume increases and the average cell size decreases by subjecting the matrix to a larger pressure decline $[14,15,18,25]$. Contrary to the foaming pressure, the effects of foaming temperature on the cell morphology have not been understood precisely. However, it has been shown that temperature heterogeneity in polymer yields uneven foam morphologies. In addition, high temperature foaming process promotes the formation of large cells with wide size distribution (compare the results of EV6EG149FT50 and EV6EG149FT70 in Table 2), whereas lower temperatures particularly those closer to the $T_{\mathrm{g}}$ of the polymer-gas solution results in the foams with possessing a relatively uniform microcellular core [15].

As can be found in Figure 4 and Table 2, different cell morphologies are attained by varying the foaming temperature above and below the EVA crystallization temperature. Similar to different graphite structures, the crystalline regions of EVA matrix are impermeable and act as physical obstacles to dissolving the nitrogen gas in polymer and consequently, increase the nucleation activation energy. As a direct result, some nonfoamed regions can observed in the micrographs of foam samples prepared below the EVA 


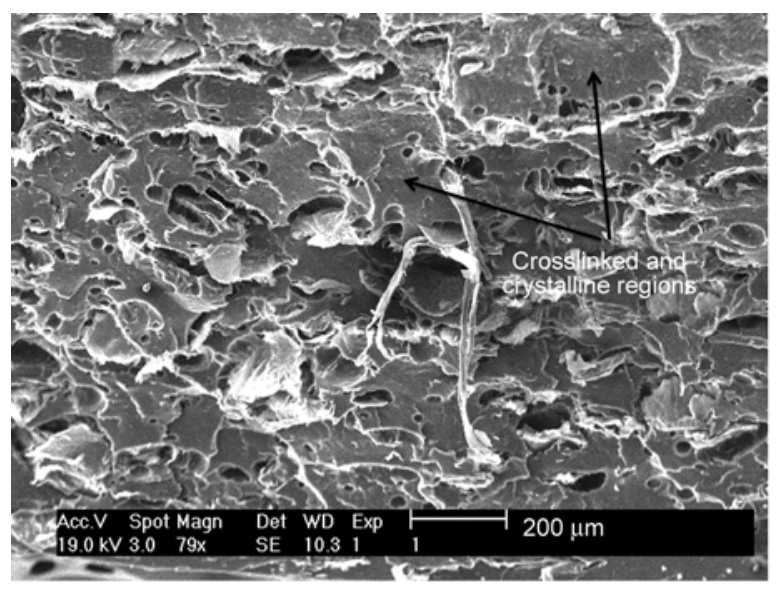

a)

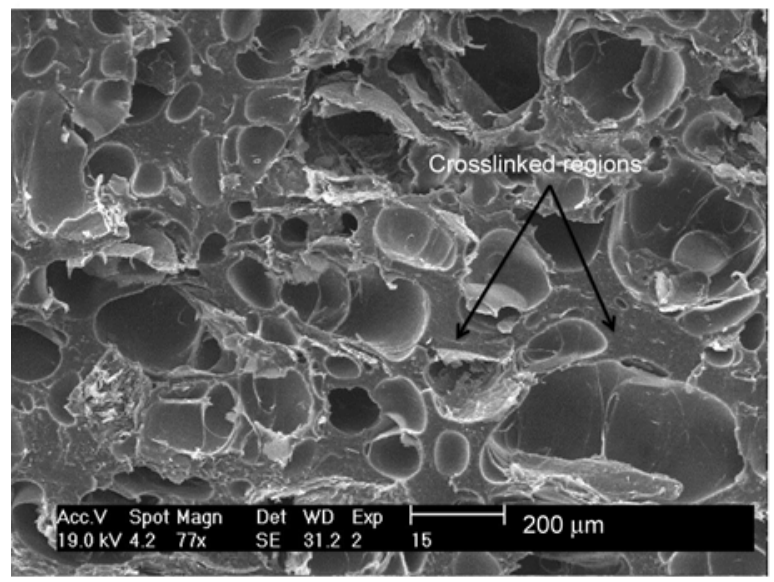

c)

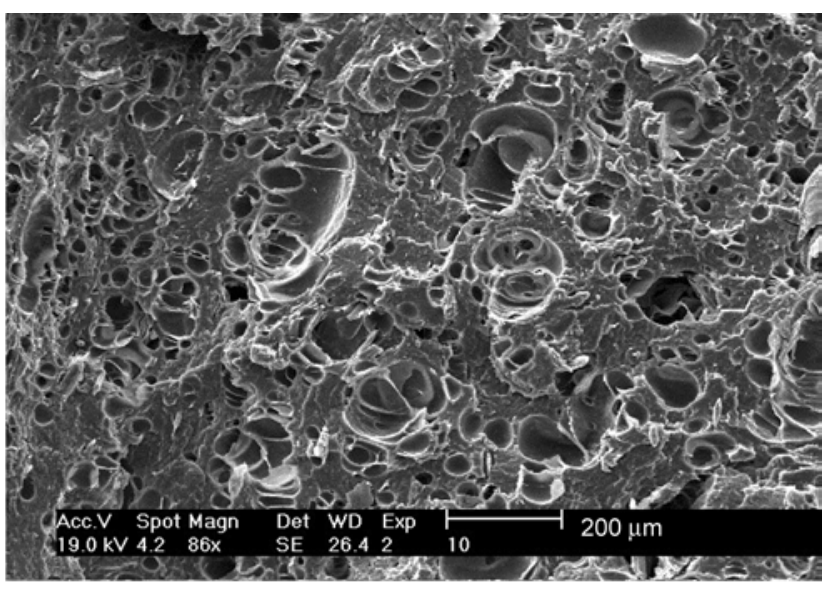

b)

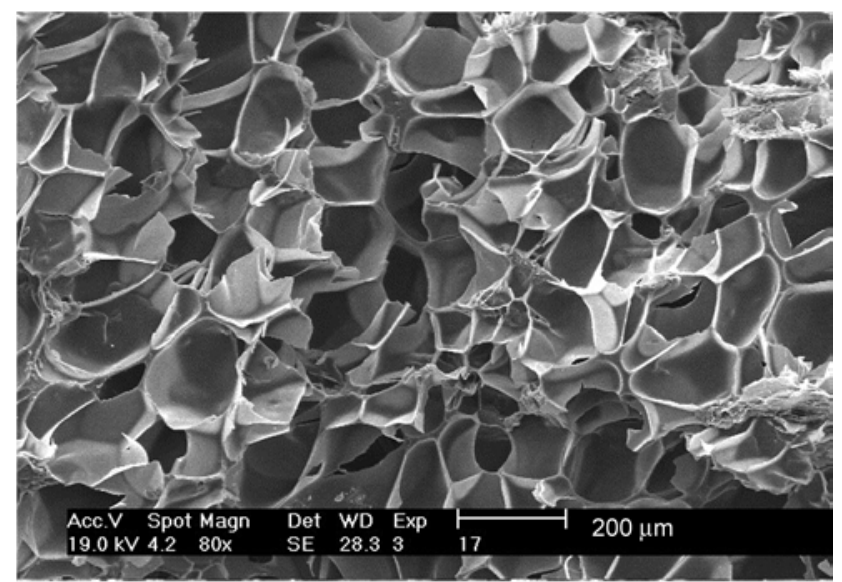

d)

Figure 4. SEM micrographs of nanocomposite foams: (a) CEV6EG149FT50; (b) EV6EG149FT50; (c) CEV6EG149FT70 and (d) EV6EG149FT70; effect of foaming temperature and crosslinking of polymer chains

crystalline meltig point (Figure $4 \mathrm{a}$ and $4 \mathrm{~b}$ ). Above the crystalline meltig point, the gas molecules have enough energy to diffuse through the polymer chains and obtain the primary bubbles. Moreover, above the crystalline meltig point the polymer chains have enough mobility to allow the bubbles to grow. Therefore, larger cells are evident in the samples foamed at higher temperature (Figure 4c and 4d). Details about the melting and crystallization behavior of EVA copolymer in the presence of expanded graphite are presented in a previous study [30].

\subsection{Void fraction}

Physical properties of polymeric foams depend mainly on three parameters: cellular microstructure or architecture, intrinsic characteristics of polymer that constitutes the solid framework and relative density or void fraction. It seems that the void fraction or relative foam density is the most notable physical characteristic of foam sample [38].

Figure 5 illustrates the effects of aforementioned parameters on the void fraction of EVA/EG nano-

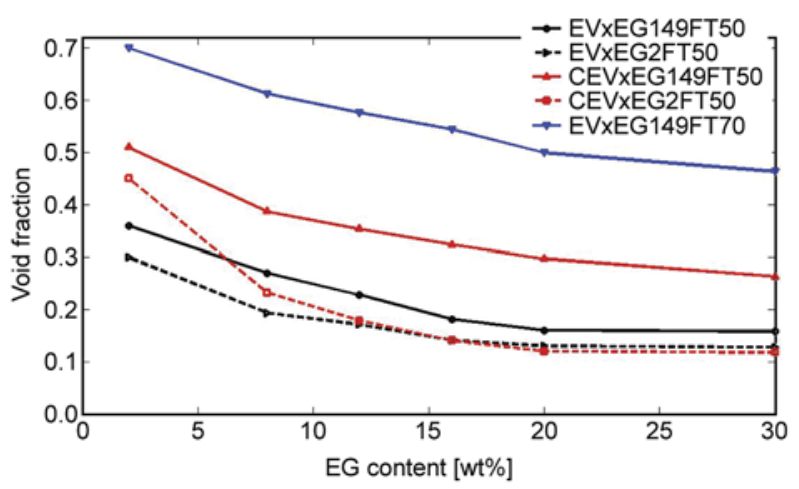

Figure 5. Void fraction versus EG content of foam samples; effect of material and processing parameters. The standard deviation for all samples is less than $3 \%$.

composite foams. For all types of these foams, the void fraction is diminished by increasing the EG loading due to the larger accessible surface area for bubble heterogeneous nucleation and correspondingly, the formation of larger quantity of smaller cells. In addition, the existence of more EG aggregates at higher loadings of nanofiller lower the nucleation efficiency. 
The nanocomposite foams containing EX100, which are prepared at the temperature above the crystallization point, show the maximum void fractions, whereas the nanocomposite foams including EX1500 that are prepared at the temperature below the crystallization temperature, have comparatively the minimum amounts of void fraction. According to the SEM micrographs, the foam sample containing EX100 with higher particle size possesses larger cell size and broader cell size distribution owing to the growth of more bubbles in the foaming process in comparison with the EX1500 incorporated foams. Another influential factor in the cell formation process particularly at lower foaming temperature is the crystallinity weight fraction of solid composites. It was found in the previous paper that the nanocomposites containing EX100 show larger degree of crystallinity than EVA/EX1500 solid samples [30]. Therefore, more crystalline regions of EVA/EX100 samples diminish the accessible domain area for bubble nucleation and growth. Furthermore, the nanocomposite foams obtained above the EVA crystallization temperature have relatively higher void fraction, which stems from large accessible matrix domains for cell nucleation and growth. One of the most striking features of Figure 5 is that for the nanocomposite foams including more than $15 \mathrm{wt} \% \mathrm{EG}$, the void fraction approximately reaches a plateau. In other words, after a certain content of EG particles, the void fraction of foam samples becomes fixed and a final plateau can be observed for highly filled nanocomposites regardless of the foam type. Seemingly, the mutual EG influences on providing heterogeneous cell nucleation sites and retarding the bubble growth in foaming process remains roughly constant at high filler loadings. Another pronounced feature of this graph is the comparatively lower void fraction of crosslinked EVA foam samples. The void fraction of these samples is significantly smaller than that of non-crosslinked EVA samples and is further supported by the morphology of these foam samples. As discussed before, the crosslinked EVA/EG foams have some crosslinked regions that are free from bubbles, thereby reducing the available matrix domains for cell formation and growth.

\subsection{Dynamic mechanical analysis of foams}

There are distinct relaxation processes for the EVA copolymer chains that relate to the crystalline phase (comprised of polymethylenic segments), the inter- facial regions (consisting of both ethylenic and VAc segments) and the complex amorphous phase (composed of noncrystallized methylenic segments and VAc units). These relaxation processes can be denoted by the respective transitions: $\alpha, \beta$, and $\gamma$, which refer to the main transition, VAc segments, and the methylenic units. In the previous study, the effects of EG particle size on the DMTA results were discussed [30]. However, in the present work, the storage and loss moduli were recorded as a function of temperature for the foam samples having unequal EG particle size and different foaming temperatures. In addition, the influence of EVA crosslinking was also evaluated as a material parameter.

\subsubsection{Effects of EG particle size}

Figure 6 shows the storage and loss moduli of the prepared foams with different particle size. From this figure and the previous research, it can be ascertained that EVA/EG foams show different dynamic mechanical behaviors in a similar way to EVA/EG solid nanocomposites having different particle size and EG contents. However, the more effective reinforcing role of EG nanofiller in EVA matrix has been achieved for the solid nanocomposites filled with higher loadings of large aspect ratio graphite. Nonetheless, the EG particle size has reverse effect on the storage modulus of the nanocomposite foams.

According to the Figure 6a, it is clear that the addition of EG to EVA matrix improves the storage modulus of virgin foam due to the reinforcing effect of EG and the formation of more uniform cells. As mentioned earlier, the void fraction of foam is a critical parameter to determine the foam properties. This is why the foam storage modulus considerably depends on the void fraction. As can be found in Figure 6a, the nanocomposite foam containing EX100 with higher void fraction has relatively lower storage modulus in the full range of studied temperature compared to the sample containing EX1500. Polymer-filler interaction and interfacial area in the solid regions of foams, cell size, cell size distribution and void fraction of foams affect the storage and loss moduli of nanocomposite foams. The concomitant influences of these factors determine the foam void fraction and correspondingly, foam moduli. From the SEM micrographs and void fraction curves, it is apparent that the nanocomposite foam filled with $6 \mathrm{wt} \%$ of EG with lower aspect ratio (EX1500) has smaller cell size and lower void fraction in comparison with the EVA 

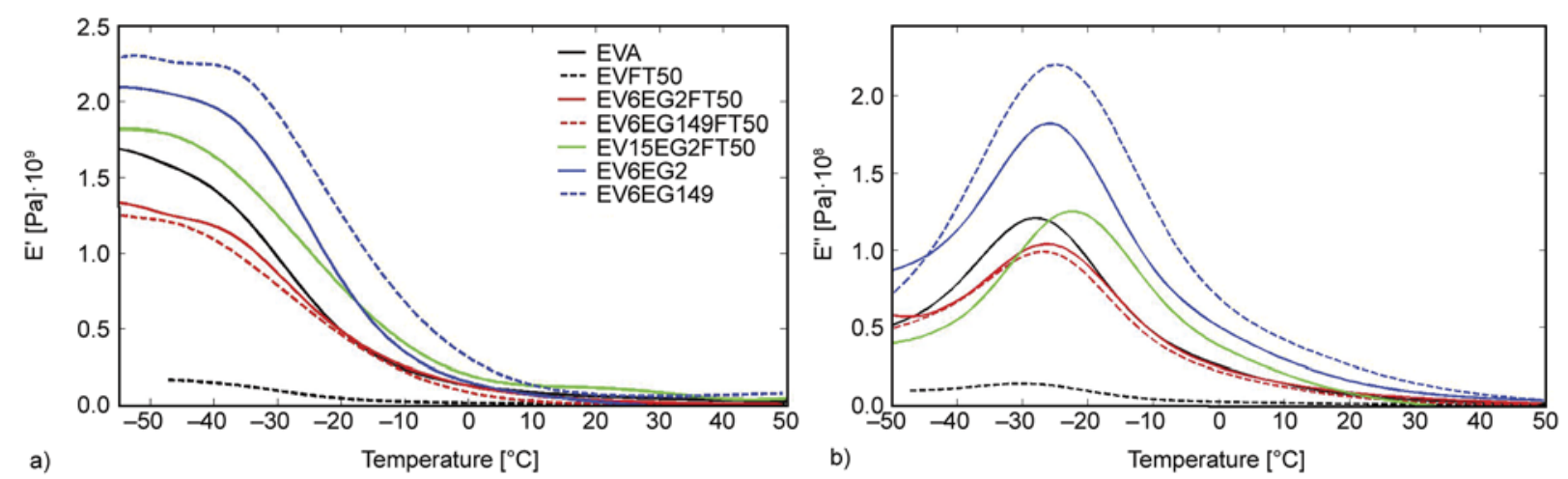

Figure 6. (a) Storage modulus and (b) loss modulus of virgin EVA, EVA foam and the solid and foamed nanocomposites with different EG particle sizes and loadings. All samples were prepared at the same conditions. The DMTA of solid nanocomposites were presented in the previous study [30].

matrix reinforced by EG with higher particle size (EX100). Seemingly, the void fraction does make a stronger impact on the foam properties than the EG aspect ratio and the dominant role of bubbles in weakening the EVA matrix is the plausible reason for the higher storage modulus of sample containing EX1500. As shown in Figure 6a, it is also obvious that the $E^{\prime}$ of foamed samples are relatively lower than the $E^{\prime}$ of solid nanocomposites due to the presence of gas phase in the foamed samples and the formation of non-uniform cell structure.

Furthermore, Figure $6 \mathrm{~b}$ demonstrates that the foaming process of polymer in the presence of EG particle changes the loss modulus curves substantially and the incorporation of EG nanofiller into EVA matrix enhances the peak position of loss modulus or the EVA glass transition temperature. The latter is caused by the slower dynamics and restricted mobility of polymer chain segments that interact with graphite sheet surfaces.

An interesting phenomenon, which is worth noting, is that the foaming process of polymer significantly decreases the value of loss modulus. Particularly for the pure EVA foam, the obtained heterogeneous structure of polymer after foaming process is most likely to diminish the storage and loss modulus.

According to the loss modulus curves of samples containing $6 \mathrm{wt} \%$ of EGs, i.e. EX100 and EX1500, the foaming process of EVA nanocomposites similarly reduces the $E^{\prime \prime}$ peak heights, indicating that the polymer chain mobility and the resultant chain damping ability is noticeably decreased by foaming the EVA even in the presence of EG particles. During the foaming process, the formation of bubbles in polymer matrix imposes major constraints on polymer chains by passing through the macromolecules and

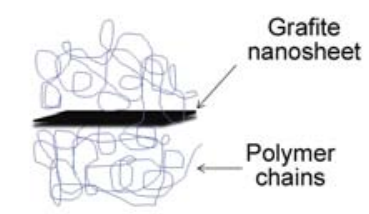

a)

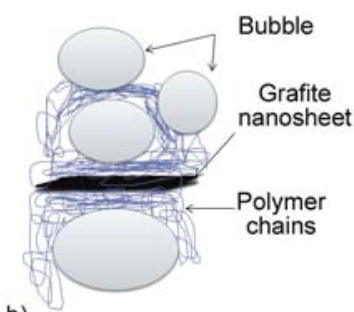

b)

Figure 7. Schematic diagrams indicating the orientation and intermolecular distance of polymer chains in: (a) solid EVA/EG nanocomposite and (b) EVA/EG foam nanocomposite; the chains are tightly packed after foaming process

diminishing the chain-to-chain intermolecular distances. Therefore, squeezing the chains into smaller spaces, orienting the polymer chain segments towards each other and increasing the total chain segments that interact with EG nanosheets are the plausible explanations of impeded EVA molecular movements after foaming and corresponding lower foam loss modulus. Figure $7 \mathrm{a}$ and $7 \mathrm{~b}$ are schematically show these phenomena. In a similar manner, this trend is also observed for the nanocomposite foams containing higher loadings of EG particles that are not shown here.

\subsubsection{Effects of EVA crosslinking}

As mentioned earlier, along with additive addition, polymer properties such as melt strength and crystallization behavior affect the foam structure, i.e. the bubble nucleation and growth stages of foaming process. As one of the key features that influences the EVA matrix characteristics, the implications of chain crosslinking for foam properties have been evaluated here. From the aforementioned discussion on the morphology and void fraction of the crosslinked foams, it was concluded that crosslink- 

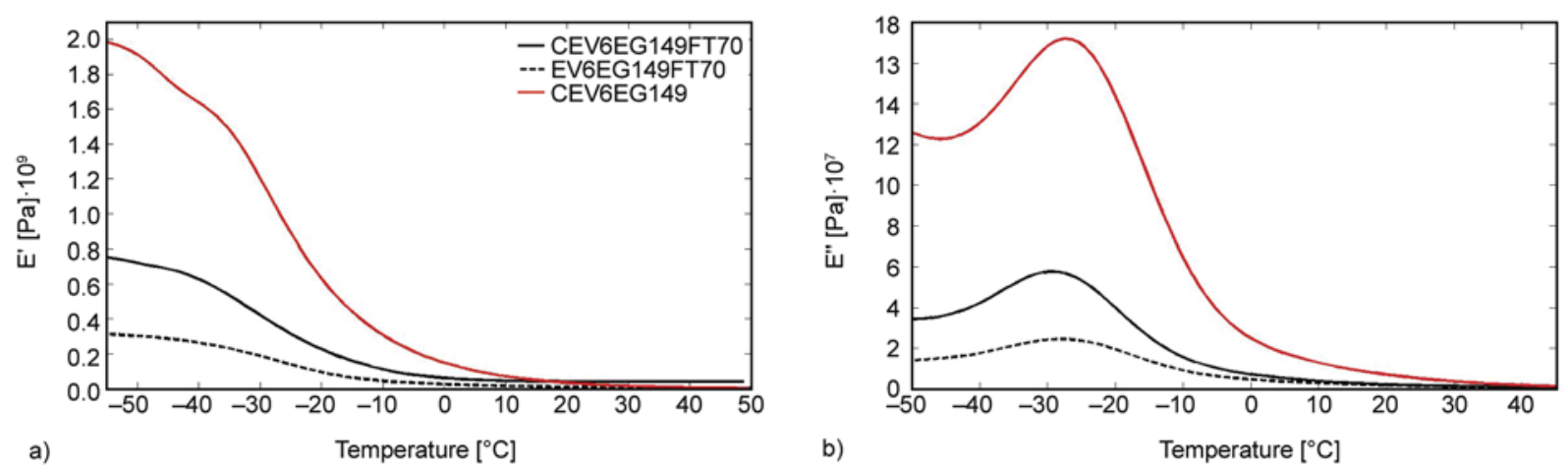

Figure 8. (a) Storage modulus and (b) loss modulus of crosslinked EVA nanocomposite and nanocomposite foams. All samples were prepared at the same conditions.

ing of polymer chains relatively reduces the foam cell density and void fraction. As it can be seen in Figure 8, crosslinked EVA foam containing $6 \mathrm{wt} \%$ of EX100 (CEV6EG149FT70) shows higher stiffness, storage and loss moduli at temperatures below the glass transition temperature compared to the noncrosslinked foam with the same composition (EV6EG149FT70). Chemical crosslinking of polymer chains changes both the matrix molecular structure and foam cell morphology. While foaming process weakens the solid crosslinked EVA/EG nanocomposite, namely CEV6EG149, crosslinking of polymer chains in the nanocomposite foam, EV6EG149FT70, enhances the EVA viscoelastic properties owing to the smaller cell density and void fraction. In the case of crosslinked samples, gas permittivity and bubble nucleation is influenced by the 3-dimensional network of polymer chains during the foaming process, thereby shifting the viscoelastic curves of foam samples.

Figure $8 \mathrm{~b}$ illustrates the loss modulus variation versus temperature for crosslinked and non-crosslinked foam samples. The height of loss modulus peak and area under this curve indicate that the total constraints imposed on EVA chains in crosslinked foam sample is lower than that of noncrosslinked one. The higher damping ability of crosslinked foam sample, CEV6G149FT70, is most probably caused by the smaller void fraction and more nonfoamed regions (refer to the schematic drawings of Figure 7).

\subsubsection{Effects of foaming temperature}

Another crucial factor that is directly involved the foam characteristics is the foaming process temperature. Besides, in semicrystalline polymers, the crystalline regions act as major obstacles for gas mole- cules to diffusing and for bubbles to growing in the solid polymer just similar to the impermeable graphite nanostructures. In this study, the nitrogen saturation temperature in the foaming process was near the melting point of EVA copolymer. Nevertheless, the foaming temperature was selected to be above and below the EVA crystallization temperature in order to make a temperature impact assessment. The melting and crystallization behavior of EVA/EG nanocomposites was investigated earlier [30]. Figure $9 \mathrm{a}$ and $9 \mathrm{~b}$ demonstrate the variation of storage and loss modulus alongside temperature for the foam samples, which were prepared at different foaming temperatures. It is clearly evident that the foam sample obtained at the temperature below the EVA crystallization point shows higher storage modulus in glassy region due to lower void fraction and the formation of special morphology that was shown in Figure 4. Similar to the cell morphologies illustrated in Figure 4, it can be deduced that the crystalline regions of the nanocomposite foamed below the EVA crystallization temperature have markedly larger area than the one for sample prepared at higher temperature. As a result of higher crystallinity degree, the EV6EG2FT50 sample shows stronger viscoelastic properties than EV6EG2FT70 foam. Moreover, the height of loss modulus peak and area under the curve of EV6EG2FT70 sample are considerably lower than that of EV6EG2FT50. As explained earlier, the foaming of EVA in the presence of EG particles decreases the loss modulus peak height because of the EVA chain squeeze, segmental orientation increase and EVA chain/EG nanolayer interface area rise. This phenomenon intensifies the interactions of EVA chains with each other and EG solid surfaces, therefore the EVA molecular movements and the peak height of loss modulus is 

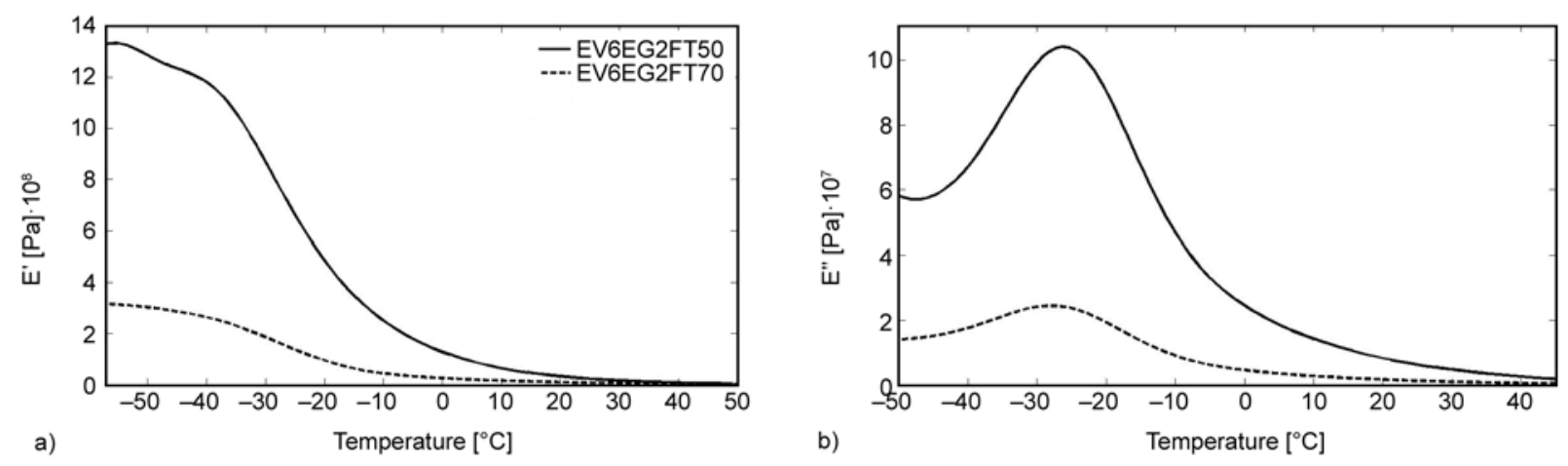

Figure 9. (a) Storage and (b) Loss modulus of EVA/EG nanocomposites foamed at different temperatures; Both samples were prepared via the same process apart from the foaming temperature.

comparatively much lower for the samples having higher void fractions.

In Figure $10, E^{\prime \prime}$ is plotted as a function of $E^{\prime}$ in the studied temperature range, which is typically known as Cole-Cole plot. The relaxation time distribution of polymer chains was described more accurately by an advanced theory proposed in the work of Cole and Cole [39]. This model represents the chain relaxation time by a depressed semi-circle. Any deviation from a single relaxation time is shown up by a more imperfect semi-circle and an extra arc at lower temperatures [40]. This extra arc refers to the dynamic heterogeneity in the system and appears at different chain relaxation time. The amplitude of the additional arc at lower temperatures is more considerable for EV6EG2FT50 and EV6EG149FT50 samples, which proves the existence of greater heterogeneity in polymer chain dynamics. This finding can give a further evidence for the restricted molecular movements of foam samples having larger void fraction. However, the mechanisms of how the addition of EG affects the relaxations of all polymer

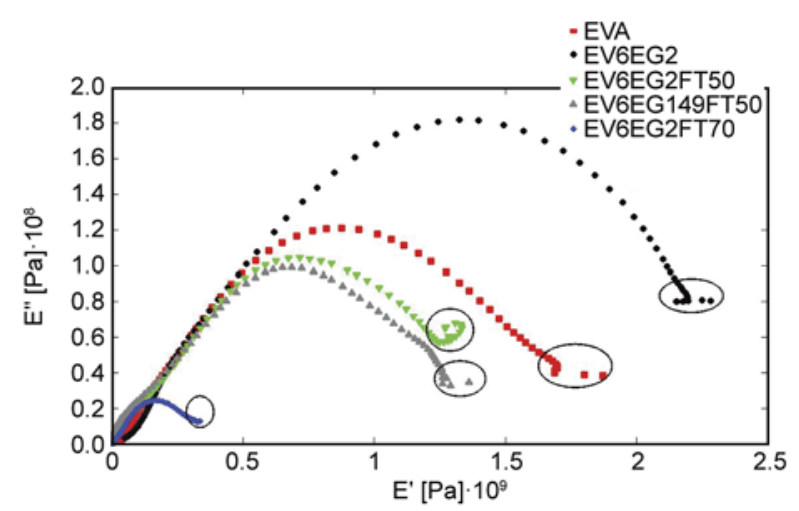

Figure 10. Cole-Cole plot for neat EVA, EVA/EG nanocomposite and EVA/EG nanocomposite foams. The ellipse indicates the aforementioned arc in the text. chains after foaming process needs to be studied further considering the profound impact of foam molecular motions on the product final properties.

\section{Conclusions}

The nanocomposite foams based on EVA/EG were made using a supercritical fluid. Different cell morphologies were obtained for the nanocomposite foams containing different EGs with unequal particle size. The solid surfaces of EG sheets act as heterogeneous nucleation sites in the foaming process and multiple cell nucleations simultaneously occur on a single nanolayer. Higher cell density and broader cell size distribution of sample filled with EX100 are the proofs of stronger multinucleations of EX100 due to the higher surface area. Furthermore, in the foam sample based on EX100, there are so many cells, which grow into one and impinge into each other. Above the EVA crystallization temperature, the gas molecules have enough energy to pass through the polymer chains, thereby producing the primary bubbles and the polymer chains themselves have quickly enough molecular movements to allow the bubbles to grow. The concomitant phenomena result in larger cell size and void fraction for nanocomposites foamed at higher temperature. The only obstacle to diffusing gas molecules and growing bubbles at this temperature is the impermeable graphite particles. Therefore, the foaming temperature plays a key role in determining the final foam properties. Besides, the void fraction decreases with increasing the EG content owing to the larger accessible heterogeneous nucleation sites and more aggregations of nanosheets at higher loadings. The nanocomposite foams containing EX100 with higher particle size prepared at the temperature above the EVA crystallization point show maximum void fraction, 
whereas the nanocomposite foams incorporating EX1500 obtained at the temperature below the crystallization point possess minimum void fraction. According to the DMTA data, the addition of EG to EVA matrix improves the foam storage and loss moduli because of the reinforcing effect of EG nanofiller and the formation of more uniform cells. In agreement with the morphology analysis and void fraction results, the nanocomposite foams with higher void fraction and larger bubble size demonstrate smaller values of both storage and loss modulus. Lower polymer chain mobility after foaming of EVA in the presence of EG particles lead to these changes. EVA/EG Foam properties are also influenced by polymer chain chemical crosslinking. More nonfoamed regions were observed in these samples due to the crosslinked regions in the EVA polymeric matrix.

\section{References}

[1] Fleming O. S., Kazarian S. G.: Polymer processing with supercritical fluids. in 'Supercritical carbon dioxide' (eds.: Kemmere M. F., Meyer T.) Wiley-VCH, Weinheim, 205-238 (2006). DOI: $10.1002 / 3527606726 . c h 10$

[2] Almanza O., Rodríguez-Pérez M. A., de Saja J. A.: The microstructure of polyethylene foams produced by a nitrogen solution process. Polymer, 42, 7117-7126 (2001).

DOI: 10.1016/S0032-3861(01)00107-0

[3] Kumar V., Weller J. E.: A process to produce microcellular PVC. International Polymer Processing, 8, 73-80 (1993).

DOI: $10.3139 / 217.930073$

[4] Krause B., Mettinkhof R., van der Vegt N. F. A., Wessling M.: Microcellular foaming of amorphous high- $T_{\mathrm{g}}$ polymers using carbon dioxide. Macromolecules, 34, 874-884 (2001).

DOI: $10.1021 / \mathrm{ma} 001291 \mathrm{z}$

[5] Okamoto M., Nam P. H., Maiti P., Kotaka T., Nakayama T., Takada M., Ohshima M., Usuki A., Hasegawa N., Okamoto H.: Biaxial flow-induced alignment of silicate layers in polypropylene/clay nanocomposite foam. Nano Letters, 1, 503-505 (2001).

DOI: $10.1021 / \mathrm{nl} 010051$

[6] Fujimoto Y., Ray S. S., Okamoto M., Ogami A., Yamada K., Ueda K.: Well-controlled biodegradable nanocomposite foams: From microcellular to nanocellular. Macromolecular Rapid Communications, 24, 457-461 (2003).

DOI: $10.1002 /$ marc. 200390068
[7] Zeng C., Han X., Lee L. J., Koelling K. W., Tomasko D. L.: Polymer-clay nanocomposite foams prepared using carbon dioxide. Advanced Materials, 15, 17431747 (2003). DOI: $10.1002 / \mathrm{adma} .200305065$

[8] Lee Y. H., Wang K. H., Park C. B., Sain M.: Effects of clay dispersion on the foam morphology of LDPE/clay nanocomposites. Journal of Applied Polymer Science, 103, 2129-2134 (2007).

DOI: $10.1002 /$ app. 24908

[9] Colton J. S., Suh N. P.: The nucleation of microcellular thermoplastic foam with additives: Part I: Theoretical considerations. Polymer Engineering and Science, 27, 485-492 (1987).

DOI: $10.1002 /$ pen.760270702

[10] Leung S. N., Park C. B., Li H.: Effects of nucleating agents' shapes and interfacial properties on cell nucleation. Journal of Cellular Plastics, 46, 441-460 (2010). DOI: $10.1177 / 0021955 \times 10369418$

[11] Zhai W., Yu J., Wu L., Ma W., He J.: Heterogeneous nucleation uniformizing cell size distribution in microcellular nanocomposites foams. Polymer, 47, 75807589 (2006).

DOI: $10.1016 /$ j.polymer.2006.08.034

[12] Zeng C., Hossieny N., Zhang C., Wang B.: Synthesis and processing of PMMA carbon nanotube nanocomposite foams. Polymer, 51, 655-664 (2010). DOI: $10.1016 /$ j.polymer.2009.12.032

[13] Spitael P., Macosko C. W., McClurg R. B.: Block copolymer micelles for nucleation of microcellular thermoplastic foams. Macromolecules, 37, 6874-6882 (2004). DOI: $10.1021 / \mathrm{ma} 049712 \mathrm{q}$

[14] Mizumoto T., Sugimura N., Moritani M., Sato Y., Masuoka $\mathrm{H}$ :: $\mathrm{CO}_{2}$-induced stereocomplex formation of stereoregular poly(methyl methacrylate) and microcellular foams. Macromolecules, 33, 6757-6763 (2000). DOI: $10.1021 / \mathrm{ma} 000443 \mathrm{n}$

[15] Siripurapu S., Coughlan J. A., Spontak R. J., Khan S. A.: Surface-constrained foaming of polymer thin films with supercritical carbon dioxide. Macromolecules, 37, 9872-9879 (2004). DOI: $10.1021 / \mathrm{ma} 0484983$

[16] Lee P. C., Wang J., Park C. B.: Extruded open-cell foams using two semicrystalline polymers with different crystallization temperatures. Industrial and Engineering Chemistry Research, 45, 175-181 (2005). DOI: $10.1021 / \mathrm{ie} 050498 \mathrm{j}$

[17] Otsuka T., Taki K., Ohshima M.: Nanocellular foams of PS/PMMA polymer blends. Macromolecular Materials and Engineering, 293, 78-82 (2008). DOI: $10.1002 / \mathrm{mame} .200700257$

[18] Nemoto T., Takagi J., Ohshima M.: Control of bubble size and location in nano-/microscale cellular poly (propylene)/rubber blend foams. Macromolecular Materials and Engineering, 293, 574-580 (2008).

DOI: $10.1002 /$ mame. 200800015 
[19] Wright Z. C., Frank C. W.: Increasing cell homogeneity of semicrystalline, biodegradable polymer foams with a narrow processing window via rapid quenching. Polymer Engineering and Science, 54, 2877-2886 (2014). DOI: $10.1002 /$ pen.23847

[20] Garancher J-P., Fernyhough A.: Expansion and dimensional stability of semi-crystalline polylactic acid foams. Polymer Degradation and Stability, 100, 21-28 (2014). DOI: $10.1016 /$ j.polymdegradstab.2013.12.037

[21] Liao R., Yu W., Zhou C.: Rheological control in foaming polymeric materials: II. Semi-crystalline polymers. Polymer, 51, 6334-6345 (2010). DOI: 10.1016/j.polymer.2010.11.001

[22] Jenkins M. J., Harrison K. L., Silva M. M. C. G., Whitaker M. J., Shakesheff K. M., Howdle S. M.: Characterisation of microcellular foams produced from semi-crystalline PCL using supercritical carbon dioxide. European Polymer Journal, 42, 3145-3151 (2006). DOI: 10.1016/j.eurpolymj.2006.07.022

[23] Ghanbar S., Yousefzade O., Hemmati F., Garmabi H.: Microstructure and thermal stability of polypropylene/ bagasse composite foams: Design of optimum void fraction using response surface methodology. Journal of Thermoplastic Composite Materials, in press (2015). DOI: $10.1177 / 0892705714535795$

[24] Bauhofer W., Kovacs J. Z.: A review and analysis of electrical percolation in carbon nanotube polymer composites. Composites Science and Technology, 69, 14861498 (2009). DOI: 10.1016/j.compscitech.2008.06.018

[25] Moniruzzaman M., Winey K. I.: Polymer nanocomposites containing carbon nanotubes. Macromolecules, 39, 5194-5205 (2006).

DOI: $10.1021 / \mathrm{ma} 060733 \mathrm{p}$

[26] Yasmin A., Luo J-J., Daniel I. M.: Processing of expanded graphite reinforced polymer nanocomposites. Composites Science and Technology, 66, 1182-1189 (2006). DOI: 10.1016/j.compscitech.2005.10.014

[27] Li J., Kim J-K., Sham M. L.: Conductive graphite nanoplatelet/epoxy nanocomposites: Effects of exfoliation and UV/ozone treatment of graphite. Scripta Materialia, 53, 235-240 (2005).

DOI: $10.1016 /$ j.scriptamat.2005.03.034

[28] Lee L. J., Zeng C., Cao X., Han X., Shen J., Xu G.: Polymer nanocomposite foams. Composites Science and Technology, 65, 2344-2363 (2005).

DOI: $10.1016 /$ j.compscitech.2005.06.016

[29] Chen L., Ozisik R., Schadler L. S.: The influence of carbon nanotube aspect ratio on the foam morphology of MWNT/PMMA nanocomposite foams. Polymer, 51, 2368-2375 (2010).

DOI: $10.1016 /$ j.polymer.2010.03.042
[30] Yousefzade O., Hemmati F., Garmabi H., Mahdavi M.: Thermal behavior and electrical conductivity of ethylene vinyl acetate copolymer/expanded graphite nanocomposites: Effects of nanofiller size and loading. Journal of Vinyl and Additive Technology, in press (2015). DOI: $10.1002 / \mathrm{vnl} .21428$

[31] Rodríguez-Pérez M. A., Almanza O., del Valle J. L., González A., de Saja J. A.: Improvement of the measurement process used for the dynamic mechanical characterisation of polyolefin foams in compression. Polymer Testing, 20, 253-267 (2001).

DOI: 10.1016/S0142-9418(00)00030-1

[32] Colton J. S., Suh N. P.: The nucleation of microcellular thermoplastic foam with additives: Part II: Experimental results and discussion. Polymer Engineering and Science, 27, 493-499 (1987).

DOI: $10.1002 /$ pen.760270703

[33] Chen L., Wang X., Straff R., Blizard K.: Shear stress nucleation in microcellular foaming process. Polymer Engineering and Science, 42, 1151-1158 (2002).

DOI: $10.1002 /$ pen.11019

[34] Han J. H., Han C. D.: Bubble nucleation in polymeric liquids. II. Theoretical considerations. Journal of Polymer Science Part B: Polymer Physics, 28, 743-761 (1990). DOI: $10.1002 /$ polb.1990.090280510

[35] Rodríguez-Pérez M. A., Simoes R. D., Roman-Lorza S., Alvarez-Lainez M., Montoya-Mesa C., Constantino C. J. L., de Saja J. A.: Foaming of EVA/starch blends: characterization of the structure, physical properties, and biodegradability. Polymer Engineering and Science, 52, 62-70 (2012).

DOI: $10.1002 /$ pen.22046

[36] Zhang B. S., Zhang Z. X., Lv X. F., Lu B. X., Xin Z. $\mathrm{X}$.: Properties of chlorinated polyethylene rubber/ethylene vinyl acetate copolymer blend-based foam. Polymer Engineering and Science, 52, 218-224 (2012). DOI: 10.1002/pen.22071

[37] Park K-W., Kim G-H.: Ethylene vinyl acetate copolymer (EVA)/multiwalled carbon nanotube (MWCNT) nanocomposite foams. Journal of Applied Polymer Science, 112, 1845-1849 (2009).

DOI: 10.1002/app.29736

[38] Alvarez P., Mendizabal A., Petite M. M., RodríguezPérez M. A., Echeverria A.: Finite element modelling of compressive mechanical behaviour of high and low density polymeric foams. Materialwissenschaft und Werkstofftechnik, 40, 126-132 (2009).

DOI: $10.1002 /$ mawe.200900417

[39] Cole K. S., Cole R. H.: Dispersion and absorption in dielectrics I. Alternating current characteristics. Journal of Chemical Physics, 9, 341-351 (1941). DOI: $10.1063 / 1.1750906$

[40] Shaw M. T., MacKnight W. J.: Introduction to polymer viscoelasticity. Wiley, Hoboken (2005). 